\title{
Multifractal properties of critical eigenstates in two-dimensional systems with symplectic symmetry
}

\author{
Ludwig Schweitzer \\ Physikalisch-Technische Bundesanstalt, Bundesallee 100, D-38116 Braunschweig, Germany
}

\begin{abstract}
The multifractal properties of electronic eigenstates at the metal-insulator transition of a two-dimensional disordered tight-binding model with spin-orbit interaction are investigated numerically. The correlation dimensions of the spectral measure $\widetilde{D}_{2}$ and of the fractal eigenstate $D_{2}$ are calculated and shown to be related by $D_{2}=2 \widetilde{D}_{2}$. The exponent $\eta=0.35 \pm 0.05$ describing the energy correlations of the critical eigenstates is found to satisfy the relation $\eta=2-D_{2}$.
\end{abstract}

PACS numbers: 71.30.+h, 64.60.Ak, 72.15.Rn 
Electronic states in disordered systems are known to be either localized or extended. At $T=0$ the so called mobility edge separates insulating (localized) from current carrying (extended) states. Recently, the electronic properties directly at the critical point have received increasing attention [1] 6]. Two-dimensional (2d) systems are very well suited for numerical investigations of the electronic eigenstates at a critical point. The only systems, however, that exhibit a complete Anderson transition in $2 \mathrm{~d}$ are models with symplectic symmetry. This is in contrast to the orthogonal and the unitary case where all states are localized (weak localization) and the quantum Hall (QHE) systems where only localized states with a diverging localization length at some singular energies can be observed.

In a recent paper, Chalker and coworkers [7] reported on numerical investigations of eigenstate fluctuations and correlations near the mobility edge of a two-dimensional tight-binding model with spin-orbit coupling. It was found that the probability amplitude distribution exhibits multifractal behavior which can be characterized by a set of generalized dimensions $D_{q}$. The fractal spatial structure of the wavefunctions also shows up in the eigenfunction correlations between states close in energy. A similar behavior was found also in the quantum Hall model [1,8 [10], i.e. a two-dimensional disordered system of non-interacting electrons where a strong magnetic field causes the localization length to diverge at the centres of the Landau bands with a universal exponent [11]. For these critical states, characteristic quantities like the generalized correlation dimension of the wavefunction $D_{2}=1.62$ [8.9], the corresponding generalized dimension of the spectral measure $\widetilde{D}_{2}=0.81$ [3], the exponent $\eta=0.38$ [1,3] governing the energy eigenfunction correlations, and $\alpha_{0}=2.3$ [9, 10] which for $-1 \lesssim q \lesssim 1$ determines the so called $f(\alpha)$-distribution are known, partly with sufficient precision. This is, however, not the case for the spin-orbit system mentioned above, mainly because of the small system sizes considered so far $(M \leq 18 a)$ [7]. The authors of Ref. [7] used a model proposed by Evangelou and Ziman [12] which represents a two-dimensional disordered electronic system with spin-orbit interaction.

In this letter, a different lattice model with symplectic symmetry is investigated for system sizes up to $150 a \times 150 a$, where $a$ is the lattice constant. It is found that $D_{2}=$ $1.66 \pm 0.05, \widetilde{D}_{2}=0.83 \pm 0.03, \eta=0.35 \pm 0.05$, and $\alpha_{0}=2.19 \pm 0.03$. Thus, within the numerical uncertainties, the proposed relations $\eta=2-D_{2}$ [13,9], $D_{2}=2 \widetilde{D}_{2}$ [3], and $\Lambda_{0}^{\text {typ }} \pi=\left(\alpha_{0}-2\right)^{-1}$ [14] hold also for the two-dimensional system with spin-orbit interaction.

The model used to calculate the eigenstates has been put forward by Ando [15. 16] to simulate two-dimensional systems in $n$-channel inversion layers on surfaces of III-VI semiconductors. The Hamiltonian describing this situation is

$$
\mathcal{H}=\sum_{m, \sigma} \varepsilon_{m} c_{m, \sigma}^{\dagger} c_{m, \sigma^{\prime}}+\sum_{m n, \sigma \sigma^{\prime}} V\left(m, \sigma ; n, \sigma^{\prime}\right) c_{m, \sigma}^{\dagger} c_{n, \sigma^{\prime}},
$$

with disorder potentials $\varepsilon_{m}$, creation $c_{m, \sigma}^{\dagger}$ and annihilation $c_{m, \sigma}$ operators of a particle at site $m$ and spin state $\sigma$, respectively. The transfer matrix elements $V\left(m, \sigma ; n, \sigma^{\prime}\right)=$ $\sigma \sigma^{\prime} V\left(n,-\sigma^{\prime} ; m,-\sigma\right)$ which are restricted to nearest neighbours only depend on whether the transfer from site $m$ to one of the nearest neighbours $n$ goes along the $x$ - or the $y$-direction. The strength of the spin-orbit interaction is determined by the parameter $S=V_{2} / V$, with $V=\left(V_{1}^{2}+V_{2}^{2}\right)^{1 / 2}$ taken to be the unit of energy, where $V_{1}$ and $V_{2}$ are the matrix elements for transitions with and without spin-flip, respectively. $V\left(m, \sigma ; n \sigma^{\prime}\right)$ is then given by 


$$
V_{x}=\left(\begin{array}{ll}
V_{1} & V_{2} \\
-V_{2} & V_{1}
\end{array}\right), V_{y}=\left(\begin{array}{ll}
V_{1} & \mathrm{i} V_{2} \\
\mathrm{i} V_{2} & V_{1}
\end{array}\right), \quad|\sigma=+1\rangle=\left(\begin{array}{l}
1 \\
0
\end{array}\right),|\sigma=-1\rangle=\left(\begin{array}{l}
0 \\
1
\end{array}\right) .
$$

The localization properties of symplectic models have previously been analyzed numerically 12,15 20 from which a metal-insulator transition can be inferred. The most recent calculations of Fastenrath [20,21] report a critical exponent $\nu=2.75$ for the localization length at the band centre, $E / V=0$, together with a critical disorder $W_{c}=5.74 \mathrm{~V}$ for a constant probability distribution of the on-site disorder potentials $\left\{\varepsilon_{m}\right\}$ and a spin-orbit strength $S=0.5$. We also take these parameters in what follows so that our new results supplement the already published data. The eigenvalues and eigenstates were calculated numerically by means of a Lanczos-algorithm for systems of size up to $L=150 a$ with periodic boundary conditions applied in both directions.

The structure of the eigenstates is analyzed in terms of the $f(\alpha)$-distribution [22,23] which completely characterizes the spatial scaling behavior of the $q$-moments of the wavefunction. In Fig. 1 the $f(\alpha)$-distribution for a particular eigenstate from the critical region near the center of the tight binding band $(E=0 \mathrm{~V})$ and a disorder strength $W=5.74 \mathrm{~V}$ is shown for several $q$-values. For comparison the corresponding parabolic approximation [24, 14] is also shown. The average over 190 eigenstates taken from the energy interval $[-0.15,0.0]$ gives a value of $\alpha_{0}=2.19 \pm 0.03$. This number represents the most probable value of the scaling exponents $\alpha_{q}=\mathrm{d} / \mathrm{d} q(q-1) D_{q}$.

Using the box-probability method the correlation dimension $D_{2}$ of the multifractal eigenstates is obtained from the scaling of the second moment $(\mathrm{q}=2)$ of the averaged boxprobability $P(q, \lambda)=\sum_{i}\left(\sum_{r \in \Omega_{i}(\lambda)}|\psi(r)|^{2}\right)^{q} \sim \lambda^{(q-1) D_{q}}$, where $\Omega_{i}(\lambda)$ is the size $l \times l$ of the $i$-th box. A power law relation is observed for $l=\lambda L$ in the range $2 a \leq l \leq L / 2$. The average over all eigenstates from the energy interval gives a correlation dimension $\left\langle D_{2}\right\rangle_{E}=1.66 \pm 0.05$ which is close to the value of 1.63 reported in [7] for smaller system sizes.

The correlation dimension of the spectral measure, $\widetilde{D}_{2}$, which is related to the temporal decay 25,3] of the maximum of a wavepacket (probability of return) built from critical eigenstates was calculated for the same energy interval. This exponent is obtained from the scaling relation of the local density of states

$$
\gamma(q, \varepsilon)=\lim _{\varepsilon \rightarrow 0} 1 / L^{2} \sum_{\mathbf{r}} \sum_{i}\left(\sum_{E \in \Omega_{i}(\varepsilon)}\left|a_{E}\right|^{2}\right)^{q} \sim \varepsilon^{(q-1) \widetilde{D}_{q}}
$$

with $a_{E}=\psi_{E}(\mathbf{r}) /\left(\sum_{E^{\prime}}\left|\psi_{E^{\prime}}(\mathbf{r})\right|^{2}\right)^{1 / 2}$. The scaling behavior is shown in Fig. 2 for $q=2$. Here, an exponent $\widetilde{D}_{2}=0.83 \pm 0.03$ is found which satisfies the relation $D_{2}=2 \widetilde{D}_{2}$ proposed previously for the 2d QHE system [3]. These results indicate that the diffusion at the mobility edge will be non-Gaussian in the long-time limit. Due to the multifractal nature of the spatial amplitude fluctuations of the critical wavefunctions and the behavior of the fractal spectral measure, the diffusion coefficient will not be a constant. A non-trivial frequency and wavevector dependence governed by an exponent $\eta$ has been observed in the QHE model [1.3] and a similar behavior was also seen for the symplectic case [7].

In Fig. 3 the correlations of the eigenstates close in energy

$$
Z\left(E, E^{\prime}\right)=\sum_{\mathbf{r}}\left|\psi_{E}(\mathbf{r})\right|^{2}\left|\psi_{E^{\prime}}(\mathbf{r})\right|^{2} \sim\left|E-E^{\prime}\right|^{-\eta / d}
$$


averaged over small energy intervals are shown for the critical eigenstates. A power law relation is observed with $\eta=0.35 \pm 0.05$. From the above results it is seen that the relation $\eta=d-D_{2}$ [13,9] also holds in the $2 \mathrm{~d}$ symplectic case.

We have to mention that our value for $\eta$ is compatible with the result for a different symplectic model obtained from the calculation of the two-particle spectral function at the mobility edge [7]. It is also in accordance with an earlier estimate by Evangelou [26], although the corresponding scaling exponent of the localization length, $\nu=1.6$, differs considerably from $\nu=2.75$ obtained by Fastenrath [20,21] for the Ando Model. However, to determine $\eta$ from the calculated finite size scaling variable at the critical point, $\Lambda_{c}$, Evangelou used the relation $\Lambda_{c}=1 /(\pi \eta / 2)$ which is only within the parabolic approximation $\left(D_{q}=2-q\left(\alpha_{0}-2\right)\right.$ 24, 14, valid for $\left.|q| \lesssim 1\right)$ equivalent to a relation proposed recently by Janßen [14], $\Lambda_{c}^{\text {typ }}=1 /\left(\pi\left(\alpha_{0}-d\right)\right)$, relating the typical finite size scaling variable at the critical point, $\Lambda_{c}^{\text {typ }}$, to the multifractal behavior of the eigenstates.

We note that our results for $\alpha_{0}, D_{2}, \widetilde{D}_{2}$, and $\eta$ are very close to those obtained for the 2d QHE model [10,3]. Although we could not observe any size dependence, the presently achieved system sizes do, however, not allow to exclude the possibility that the values actually coincide in the thermodynamic limit, $L \rightarrow \infty$. A similar correspondence has recently been asserted for the energy level statistics [6] of the critical 3d Anderson model with and without a magnetic field. In addition, the critical exponent of the localization length was reported to be identical in both cases [27,28]. If these observations for $3 \mathrm{~d}$ models were correct and if also the values obtained from the multifractal analysis in $2 \mathrm{~d}$ for the quantum Hall systems were indeed the same as those reported above for the symplectic disordered systems, then the behavior at the critical point would primarily be determined by the euclidean dimension and not by the symmetry class of the Hamiltonian.

In conclusion, the multifractal properties of the electronic eigenstates at the metalinsulator transition of two-dimensional disordered systems with symplectic symmetry have been investigated. The $f(\alpha)$-distribution, the correlation dimensions of the spectral measure and the critical eigenstates, and the energy correlations of the wavefunctions were calculated. The obtained values for $\alpha_{0}=2.19 \pm 0.03, \widetilde{D}_{2}=0.83 \pm 0.03, D_{2}=1.66 \pm 0.05$, and $\eta=0.35 \pm 0.05$ appear to be independent of system size and satisfy general relations that have been proposed previously for the critical states at the metal-insulator transition in other disordered systems.

\section{ACKNOWLEDGMENTS}

I gratefully acknowledge helpful discussions with Bodo Huckestein and Martin Janßen. 


\section{REFERENCES}

[1] J. T. Chalker and G. J. Daniell, Phys. Rev. Lett. 61, 593 (1988).

[2] B. I. Shklovskii et al., Phys. Rev. B 47, 11487 (1993).

[3] B. Huckestein and L. Schweitzer, Phys. Rev. Lett. 72, 713 (1994).

[4] V. E. Kravtsov, I. V. Lerner, B. L. Altshuler, and A. G. Aronov, Phys. Rev. Lett. 72, 888 (1994).

[5] T. Brandes, L. Schweitzer, and B. Kramer, Phys. Rev. Lett. 72, 3582 (1994).

[6] E. Hochstetter and M. Schreiber, Phys. Rev. Lett. 73, 3137 (1994).

[7] J. T. Chalker, G. J. Daniell, S. N. Evangelou, and I. H. Nahm, J. Phys.: Condens. Matter 5, 485 (1993).

[8] B. Huckestein and L. Schweitzer, in High Magnetic Fields in Semiconductor Physics III: Proceedings of the International Conference, Würzburg 1990, edited by G. Landwehr (Springer Series in Solid-State Sciences 101, Springer Verlag, Berlin, 1992), p. 84.

[9] W. Pook and M. Janßen, Z. Phys. B 82, 295 (1991).

[10] B. Huckestein, B. Kramer, and L. Schweitzer, Surf. Science 263, 125 (1992).

[11] B. Huckestein, Phys. Rev. Lett. 72, 1080 (1994).

[12] S. N. Evangelou and T. A. L. Ziman, J. Phys.: Condens. Matter 20, L235 (1987).

[13] J. T. Chalker, Physica A 167, 253 (1990).

[14] M. Janßen, International Journal of Modern Physics B 8, 943 (1994).

[15] T. Ando, Surf. Science 196, 120 (1988).

[16] T. Ando, Phys. Rev. B 40, 5325 (1989).

[17] A. MacKinnon, in Localization, Interactions and Transport Phenomena, edited by B. Kramer, G. Bergmann, and Y. Bruynseraede (Springer Verlag, Berlin, 1985), p. 90.

[18] A. MacKinnon, in Localization and Confinement of Electrons in Semiconductors, Vol. 97 of Springer Series in Solid State Sciences, edited by F. Kuchar (Springer Verlag, Berlin, 1990), pp. 111-116.

[19] U. Fastenrath et al., Physica A 172, 302 (1991).

[20] U. Fastenrath, Helvetica Physica Acta 65, 425 (1992).

[21] U. Fastenrath, Physica A 189, 27 (1992).

[22] T. C. Halsey et al., Phys. Rev. A 33, 1141 (1986).

[23] A. Chhabra and R. V. Jensen, Phys. Rev. Lett. 62, 1327 (1989).

[24] M. E. Cates and T. A. Witten, Phys. Rev. A 35, 1809 (1987).

[25] R. Ketzmerick, G. Petschel, and T. Geisel, Phys. Rev. Lett. 69, 695 (1992).

[26] S. N. Evangelou, J. Phys. A: Math. Gen. 23, L317 (1990).

[27] T. Ohtsuki, B. Kramer, and Y. Ono, J. Phys. Soc. Jpn. 62, 223 (1993).

[28] M. Hennecke, B. Kramer, and T. Ohtsuki, Europhys. Lett 27, 389 (1994). 


\section{FIGURES}

FIG. 1. The $f(\alpha(q))$-distribution function of critical eigenstates calculated for a system of size $L / a=150$ and $q= \pm 3, \pm 2, \pm 1.5, \pm 1, \pm 0.8, \pm 0.5, \pm 0.3,0$. The full curve is a fit using the parabolic approximation with $\alpha_{0}=2.19$.

FIG. 2. The scaling of the spectral measure at the mobility edge for a system of size $L / a=100$ from which a correlation exponent $\widetilde{D}_{2}=0.83 \pm 0.03$ is obtained.

FIG. 3. The energy correlation $Z\left(E, E^{\prime}\right)$ of the critical eigenstates as a function of energy separation showing a power law relation $\sim\left|E-E^{\prime}\right|^{-\eta / 2}$ with an exponent $\eta=0.35 \pm 0.05$. 


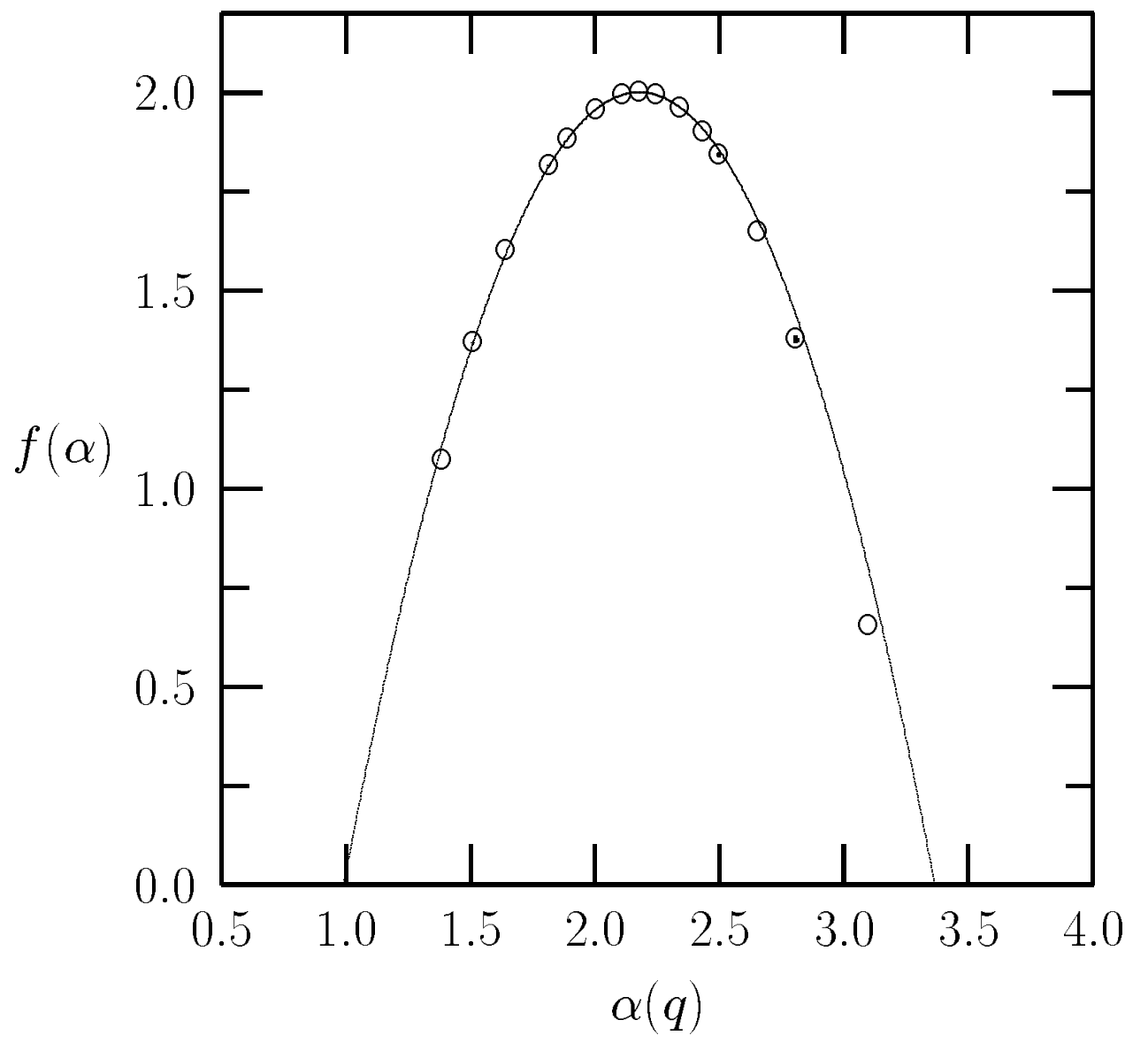




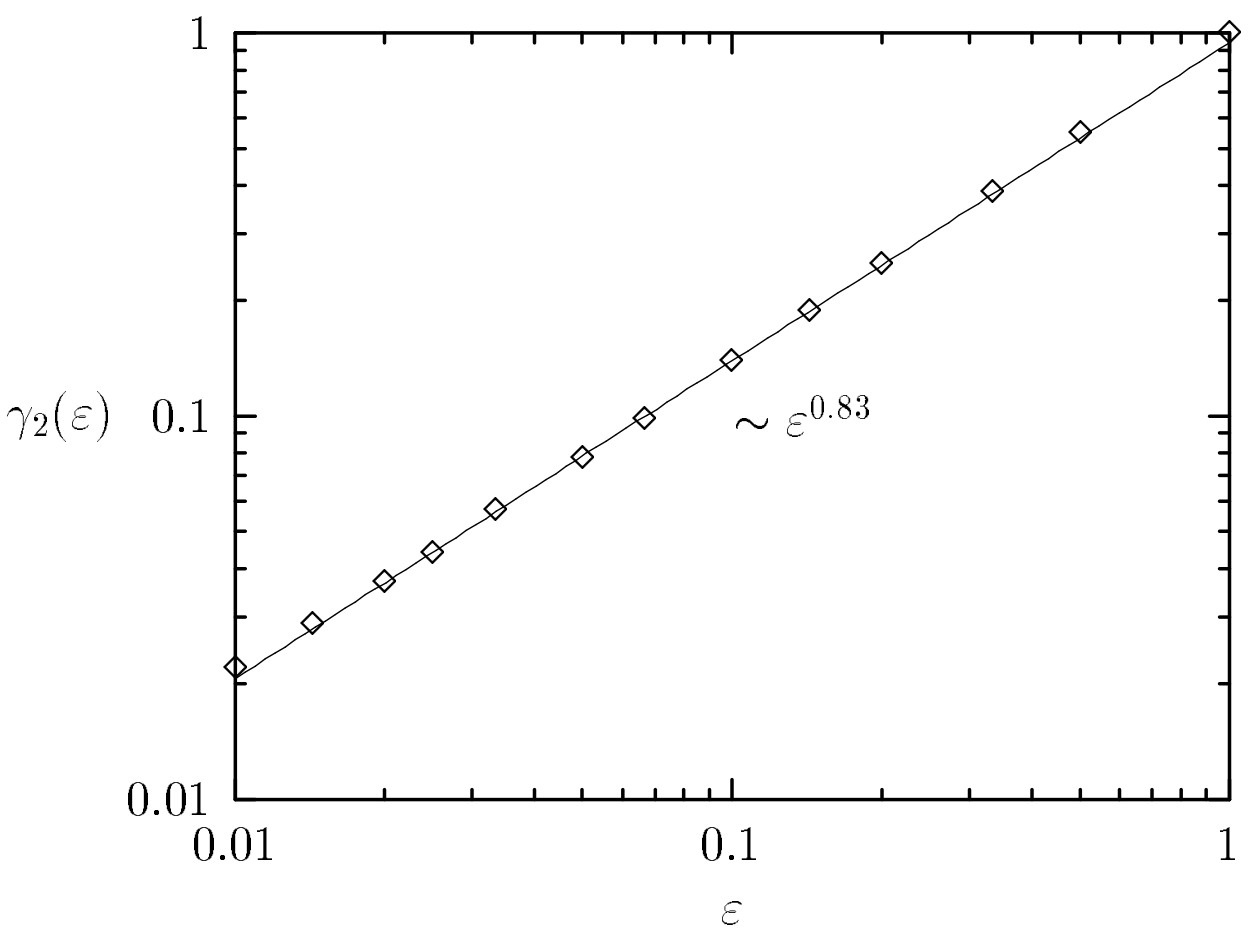




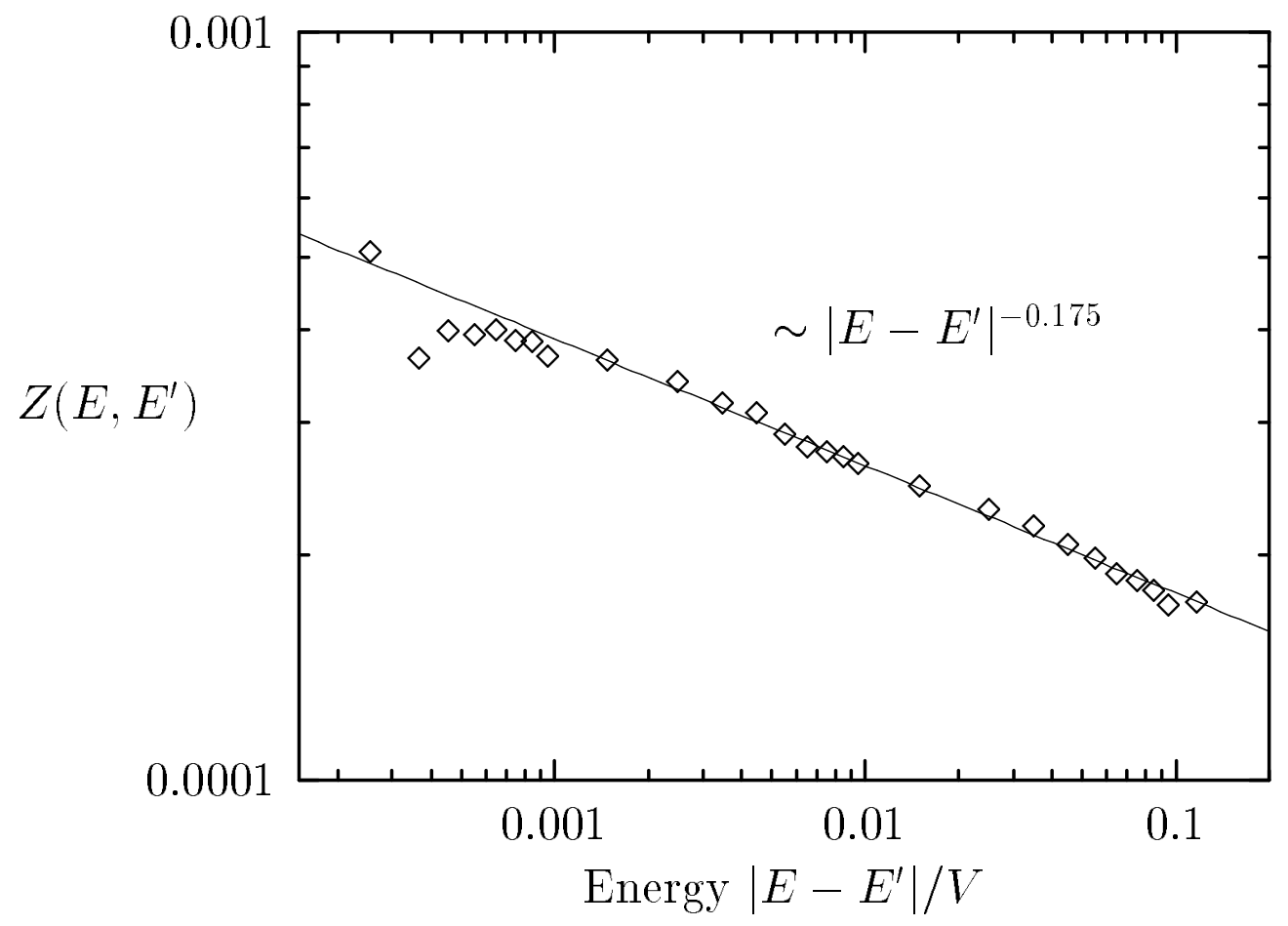

